\title{
Los riegos de oportunidad de Santolaria de Galligo/ Santa Eu- lalia de Gállego. (Estudio de caso de una gestión eficiente de un bien escaso y común)
}

\author{
Àngels Castellarnau Visús ${ }^{1}$ y José Antonio Cuchí Oterino² \\ ${ }^{1}$ Escuela Técnica Superior de Arquitectura de Barcelona, Universitat Politècnica de Catalunya y PERIFERIA. \\ arquitectura y sostenibilidad s.c.p. ${ }^{2}$ Escuela Politécnica Superior de Huesca, Universidad de Zaragoza. \\ acv@periferia.com.es, cuchi@unizar.es
}

\begin{abstract}
Resumen. Santolaria, tradicionalmente con una población superior a la soportable por su territorio, tiene escasa superficie cultivable que se destina a la auto subsistencia. La agricultura de regadío se reduce a pequeños huertos familiares en los barrancos cercanos. El déficit hídrico de finales de la primavera y durante el verano ha obligado a los habitantes a desarrollar estrategias para corregir las carencias de agua y conseguir la habitabilidad en su territorio. Así, los huertos de "monte" son una solución modesta, complementaria y eficiente, que almacena el agua de escorrentías de laderas y caminos. Ésta estructura de gestión de un bien común permite obtener un reparto igualitario del recurso basado en una estructura social de tendencia horizontal que asegura la subsistencia de la comunidad. El presente trabajo se centra en los denominados "huertos del Coronazo" para estudiar y cartografiar sus estructuras, definir sus límites, caudales y eficiencias hídrica y social. Además analiza la gestión del recurso, su elasticidad histórica y su carácter identitario.
\end{abstract}

Palabras clave: Regadíos atípicos, Gestión de un bien común, Habitabilidad, Santolaria de Galligo, Aragón.

\begin{abstract}
Santolaria, with a population exceeding the territory's capacity, has a little arable land used to grow subsistence crops. Irrigated agriculture is reduced to small family orchards placed in nearby ravines. Late spring and summer deficit of water has forced people to develop strategies to achieve the liveability on the region. Mountain irrigation systems are a modest and efficient contribution that harvests water from roads and hillsides. This common good management system provides an equitable resource sharing based on a horizontal social structure and ensures the community's survival. The present work focuses on the "Coronazo's orchards" to map their structures, identify their limits, flows and water and social efficiency. Moreover it analyzes the resource's management, its historical elasticity and its local character.
\end{abstract}

Keywords: Small scale irrigation, common pool resources management, habitability, Santolaria de Galligo, Aragón.

\section{Introducción}

Durante las últimas décadas se ha realizado un importante esfuerzo académico en el estudio de sistemas de regadío calificables como tradicionales, antiguos o históricos en la fachada mediterránea de la península Ibérica (Glick, 1968; Monter y Benito, 1986; Laliena, 1994; Piedrafita, 1995 y Cuchí, 2006). En su mayoría, los trabajos mencionados tratan sobre sistemas de riego por derivación de agua desde cursos superficiales. Menos estudiados en este territorio son los sistemas de riego asociados con las prácticas de captación de escorrentías, conocidas comúnmente como sistemas de cosecha de agua o water harvesting. Estas han sido estudiadas, por ejemplo, en el Norte de África y este de Asia por Evanari et al (1971) y Oweis et al (1999) y en el África subsahariana por Ngigi (2003). También se han citado en el sur de España (Frot et al, 2008). 
El presente trabajo se centra en el estudio de un pequeño sistema de riego localizado, que se alimenta mediante un sistema de captación de agua de escorrentías de monte situado en Santolaria de Galligo/Santa Eulalia de Gállego, provincia de Zaragoza.

\section{Marco del estudio}

Santolaria de Galligo es una pequeña localidad enclavada en la comarca de la Hoya de Huesca (Figura 1) y pertenece a la provincia de Zaragoza. Su término municipal, unos $30 \mathrm{~km}^{2}$, está situado en las estribaciones prepirenaicas, en la orilla derecha del río Gállego. Salvo una modesta superficie de terrazas fluviales, el resto del término tiene un relieve accidentado a la vista del frente de cabalgamiento pre pirenaico, orlado por los espectaculares mallos de Riglos y de Murillo. La geología inmediata está formada por una alternancia horizontal de capas de margas y areniscas calcáreas acarreadas por desaparecidos ríos pirenaicos que fluyeron en el Mioceno inferior, conformando un abanico aluvial, con ápice en Biel que recubrió parte de la entonces cerrada depresión del Ebro. La apertura de esta al mar produjo un importante proceso de erosión que encajó el río Gállego entre Riglos y Gurrea de Gállego, en un tramo relativamente natural que se pretende embalsar desde hace unos 30 años.

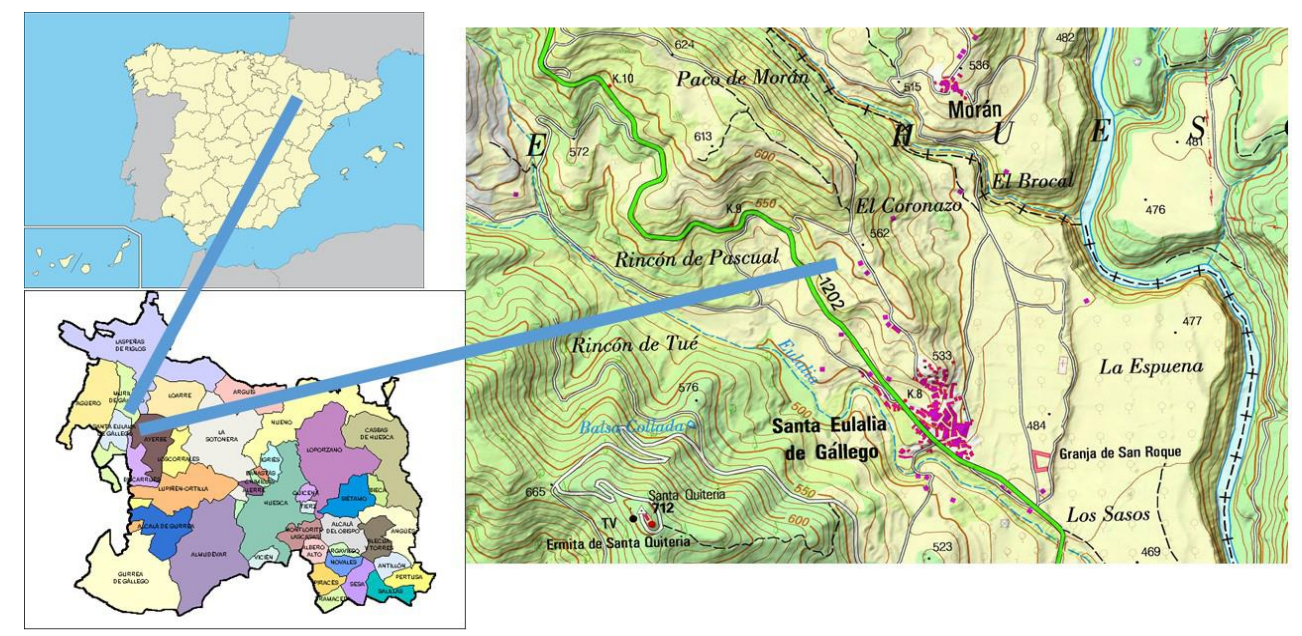

Fig. 1. Ubicación de los riegos del Coronazo en Santa Eulalia de Gállego, Zaragoza.

El clima de Santolaria es mediterráneo continental. La precipitación media anual ronda los $600 \mathrm{~mm}$, con dos etapas de precipitaciones en primavera y otoño; un invierno relativamente seco, libre de nieblas y un cálido verano también seco con irregulares tormentas de tipo convectivo. Periódicamente se suceden episodios de sequía como en la década de los 40 del siglo XX, especialmente el año 1949 conocido localmente como el año sin cosecha. La temperatura media está cercana a los $11^{\circ} \mathrm{C}$. La evapotranspiración anual se estima entorno a los $800 \mathrm{~mm}$. La vegetación natural es también de tipo Mediterráneo. Predomina el pino carrasco o blanco (P. halepenesis) acompañado de tres robles, coscoja (Q. coccifera), carrasca (Q. ilex) y chaparro (Q. cerroides), otros dos pinos, carcallo (P. pinaster) y negro (P. sylvestris) así como diversos arbustos entre como boj, lentisco, madroño, enebros y sabinas. Los campos abandonados están colonizados por aliagas (Genista scorpius) y artos (Prunus spinosa). Los cultivos son también evidentemente mediterráneos. En el pasado, se cultivaba trigo en los llanos aluviales y una miríada de pequeñas fajas. Hoy la superficie de cereal trigo en el pasado hoy sobrepasado por la cebada. Almendro, viña, y olivo, sobre todo los dos últimos, tuvieron 
importancia en el pasado y hoy repuntan tímidamente. Hoy, su agreste territorio tiene un claro uso forestal, una reducida superficie pastable y una limitada productividad agrícola.

Históricamente, Santolaria se localizó y tuvo un probable origen en la extremadura aragonesa del siglo XI. Alberga dos ermitas con arcos apuntados que se presumen góticos. Aldea de Murillo de Gállego hasta 1850, diversos indicios hacen pensar que en este momento se inició un incremento de población hasta alcanzar el millar de habitantes hacia 1920. El exceso de población y lo reducido de la superficie cultivada desigualmente repartida propició el inicio de una emigración permanente potenciada por trágicos sucesos durante la Guerra civil y acentuada por la crisis del mundo rural altoaragonés de la década de 1960. En la actualidad el censo roza las 120 personas inscritas.

Durante la época de sobrepoblación, una dura economía de auto subsistencia se organizaba entorno a la casa como unidad familiar. El aterrazamiento de empinadas laderas, la ganadería de caprino, el carboneo y la caza furtiva fueron estrategias para el autoabastecimiento necesarias, junto con una deseada pero insuficiente agricultura de regadío, reducida a unos pequeños huertos familiares.

Las escarpadas orillas del Gállego, y sus importantes avenidas, no permiten el riego en sus riberas, por lo que se habilitaron zonas de huertos regados en las orillas de los barrancos locales (Morán, Retito, Ayerbe), alguno a varios kilómetros de distancia y más de una hora de recorrido. En estos barrancos, de limitados caudales, se utilizaba el quinquinflón, palanca de elevación de agua con un contrapeso que se manejaba a mano para sacar agua de pozas naturales o artificiales. Bastantes casas disponían de varios huertos repartidos por diferentes zonas. Esta estrategia sugiere una optimización en los desplazamientos y un escalonamiento en el cultivo, asociada a la existencia de un fuerte déficit hídrico durante el verano y principios del otoño.

Una alternativa al riego convencional junto a los cauces son los "riegos de monte", solución modesta pero inteligente, con elevada eficiencia en el uso del recurso, que almacena el agua de escorrentías superficiales incluidas las de algunos tejados. En la periferia inmediata del núcleo urbano se encuentran diversas balsas de este tipo, todas de modestas dimensiones. La mayor parte se presentan de forma aislada, excepto en la zona del Coronazo, localizada en la zona superior del municipio, donde existe un sistema organizado de captación de agua. Ésta estructura de gestión de un bien común permite el reparto de agua simultáneo en todo el sistema. Las balsas se llenan de forma secuencial, devolviendo el agua sobrante al camino colector, de manera que se obtiene un reparto homogéneo e igualitario basado en una estructura social de tendencia horizontal que permite la subsistencia del conjunto de la comunidad.

Por su proximidad, aspecto y huellas de barrenos que se observan en algunos afloramientos de roca, se puede suponer que al menos parte de los actuales huertos se han instalado sobre una zona de extracción de piedra. Tras el abandono de esta actividad y sobre la roca desnuda, empezaron a elevarse una serie de cercados colocados a lo largo de la pendiente y de un camino vecinal que unía Santolaria con Fuencalderas y Biel amén del arranque de un sendero hacia Morán, cercana localidad pero barrio de Murillo de Gállego. Éstos cercados se construyen con piedra seca del mismo sitio y aprovechan la topografía del lugar para frenar tierra y drenar el agua. En el interior de estos cercados, de dimensiones similares, aparecen una serie de balsas excavadas todas en la veta de la roca arenisca. Una primera hipótesis sería que estos cercados sirvieron en su origen como corrales. Esto explicaría la forma y altura de los cercados, la cota de las balsas, la forma y talla de los caminos de acceso al conjunto, las 
edificaciones integradas en el complejo, la posición estratégica respecto a la vertiente sur y los vientos dominantes que aseguraban la sanidad a la vez que el cobijo, la posición respecto al camino y la absoluta infertilidad del suelo al tratarse de roca pura.

Por entrevistas realizadas a personas de edad del municipio, se sabe que éste nunca contó con grandes rebaños sino que más bien contó con rebaños compuestos por la agrupación de las cabezas de diversas familias destinadas a su propia subsistencia (dula). El aporte posterior de tierras, sumado al uso de corrales seria el origen de la tierra de cultivo. En cualquier caso, el origen del conjunto, tanto si se destinó desde un inicio a huertos como si hubo una fase previa de corrales, está aparejado a la construcción de una serie de estructuras de recolección y almacenamiento de aguas provenientes del monte inmediatamente superior.

\section{Descripción del sistema}

El sistema de riegos del Coronazo consiste en una serie de huertos alineados a lo largo del antiguo camino de Santolaria a Fuencalderas y Biel que seguía un cordal en dirección noreste hacia Las Gordas, San Marín y Sierra Mayor, divisoria de aguas con la cuenca del Arba de Biel.

El sistema es parte de una serie de recintos y edificaciones periurbanas que se presenta en la figura 2. La superficie total no sobrepasa la hectárea de superficie. En principio se han considerado como huerto aquellos recintos que disponen de balsa y aquellos que sin mostrarla en la actualidad han sido identificados como tales por testigos. Aproximadamente la mitad se encuentran hoy actualmente en uso. La mayor parte de los huertos cuentan con una balsa de almacenamiento. El riego se realiza de forma manual, extrayendo el agua de las balsas mediante el quinquinflon, y vertiéndola en algunos casos en una pilón de piedra (fig 8), pero la distribución habitual se realiza mediante cubos, localmente conocidos como pozales. La zona inferior del sistema cuenta ahora con agua de la red municipal de abastecimiento. Estos huertos son huertos de invierno y primavera, más soleados que los huertos situados junto al cauces de barrancos. La incertidumbre sobre el volumen de agua disponible limitaba los cultivos de final de verano y principios del otoño. Se cultivan cultivos de invierno como coles y espinacas; en la actualidad domina la patata que se siembra temprana, entorno al inicio del mes de abril. Cebollas, lechugas, borrajas, bisaltos, pepinos, melones, calabacines y calabazas se plantan en pequeñas tablas. Muchos de los huertos también tienen pequeños semilleros. Se evita implantar cultivos tardanos, como tomate que pudieran sufrir por falta de agua. Tampoco hay frutales con la excepción de membrilleros junto a las balsas y algunas parras, manzanos y almendros. Los huertos se organizan en tres grandes grupos, como se observa en la figura 3. 


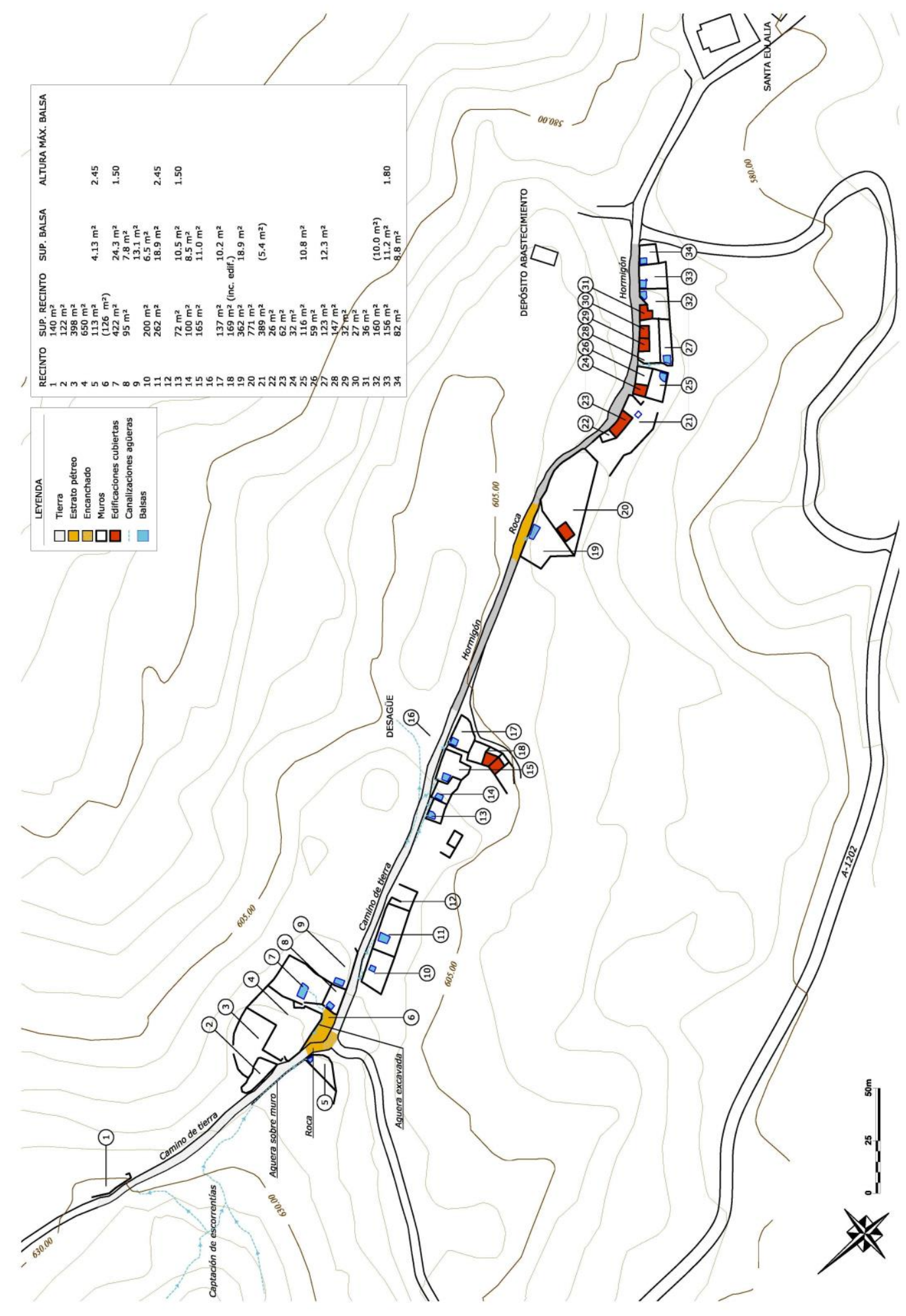

Fig. 2. Ubicación y descripción de los recintos existentes en el sistema de riego del Coronazo. 


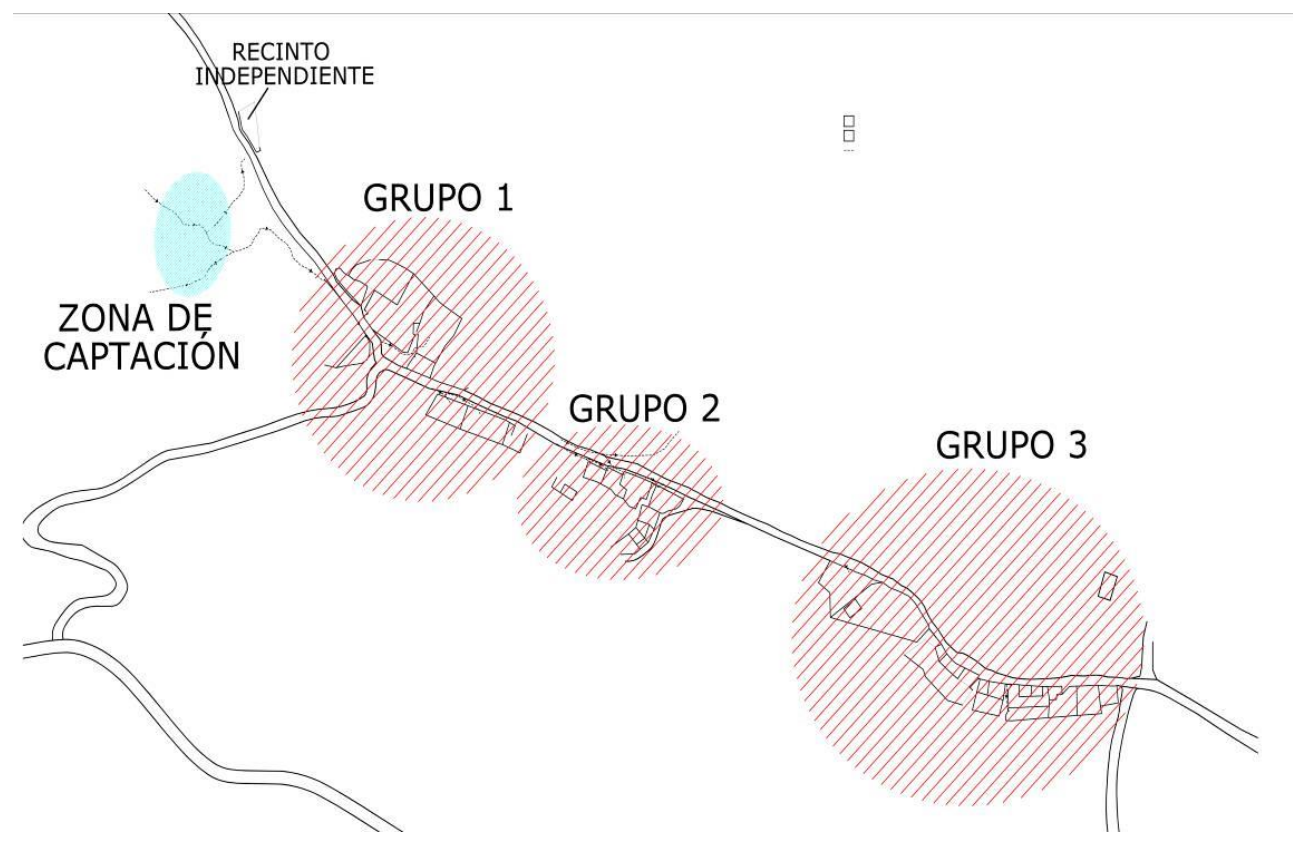

Fig. 3. Esquema grupos de huertos

Existe un primer recinto (recinto independiente) en una cota superior y justo en el cambio de vertiente de la ladera, que contaba con una desviación individual desde el sistema de captación y en el que no se ha encontrado balsa. Hoy se encuentra abandonado de todo uso.

Camino abajo, se distinguen tres grupos de huertos. El primero empezando por la parte superior recibe el agua a partir de una agüera, cuenta con un tramo elevado en un acueducto de tejas que vierte al camino y en este punto aflora la roca. La agüera continúa excavada en parte en la roca y alimenta huertos a ambos lados del camino. Unos recintos ubicados en la parte superior en los que no se han encontrado balsas, en caso de haber existidos éstas, habrían contado con una desviación anterior de la agüera previa a la entrada de ésta en el acueducto. Por testimonios orales, el recinto 7 se ha formado por unión de otros dos. En el recinto 9 no se ha encontrado toma desde la agüera del camino, puede haber desaparecido o puede que la balsa se llenase con el sobrante de la balsa del recinto 8 reforzando el carácter comunitario de la gestión del agua. Existen en el camino en este punto trazas de cantería que indican el aprovechamiento de este afloramiento para la extracción de material para la construcción de muros y edificaciones en el municipio.

En el segundo grupo de huertos las tomas se abren a la agüera que transcurre por los lados del camino, y se abastecen del agua sobrante del primer grupo sumada al agua recogida por el camino. De esta manera los usuarios se aseguran por un lado de que no se desperdicia ninguna porción del recurso y por otro lado que todas las balsas se llenas a la vez promoviendo el reparto equitativo del recurso y evitando las jerarquías en el sistema.

En el tercer grupo de huertos existen más edificaciones. Éstas están alineadas al camino y los huertos se sitúan en una segunda fila. Este grupo de huertos en la actualidad cuenta con agua de la red municipal. El recinto 20 era un antigua era, ahora convertida en huerto regado con agua de la red. El recinto 21 dispuso de balsa, pero ahora está enterrada. 
Las huertas disponen de vallado, del orden de 1,5 metros, para impedir la entrada de ganados, especialmente el caprino. Las vallas de piedra seca muestran, en general, dos niveles de obra, la inferior es de mejor calidad. En el pasado se complementaba la protección de las vallas con haces de leña. Algunos huertos cuentan con edificaciones, estas se encuentran a cierta distancia del camino, lo que podría indicar una ocupación de una vía pública. Por otro lado, se encuentran huellas de extracción de piedra en varios lugares parece indicar que una parte del espacio ocupado hubiera sido cantera en el pasado, de modo que algunos huertos hubieran sido construidos sobre canteras amortizadas. El hecho de que el nivel actual del suelo esté recrecido en varias parcelas con respecto al camino adyacente, hace pensar en un suelo de nivelado artificial, mediante el aterrazamiento y la correspondiente aportación de tierra y materia orgánica de los corrales de los propietarios como se presenta en la figura 4.

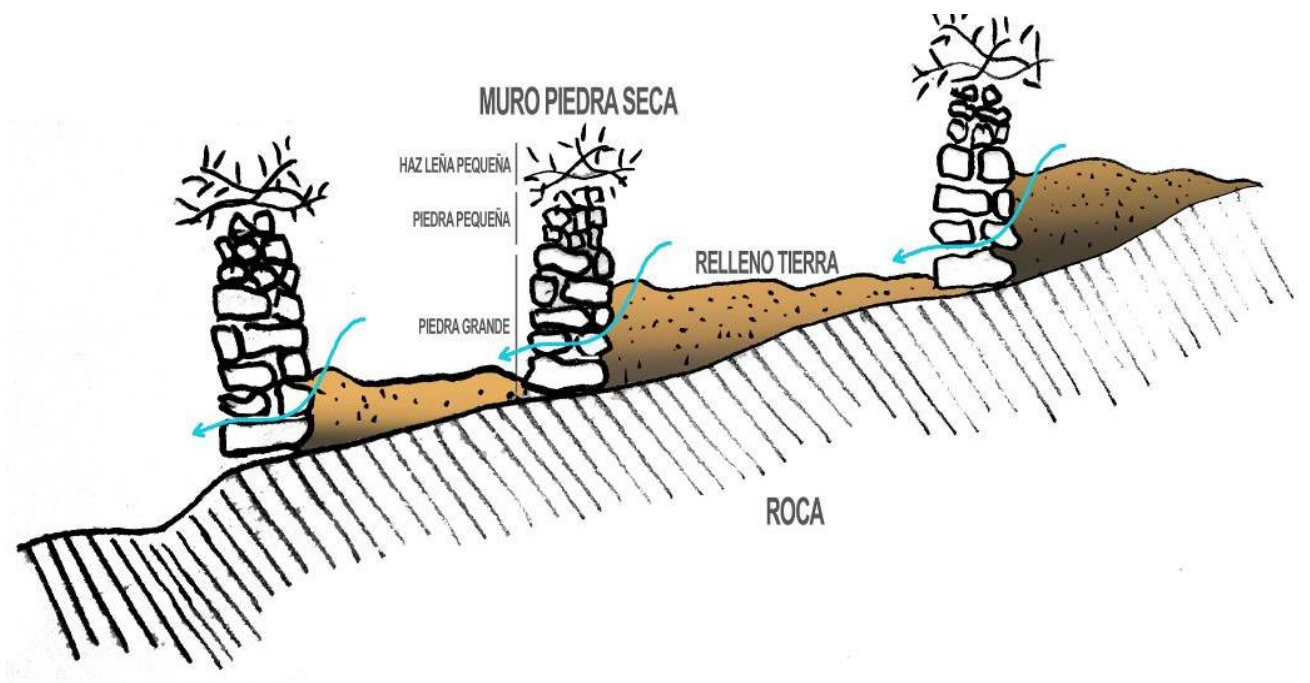

Fig. 4. Esquema formación de relleno huertos

Los huertos se organizan alineados preferentemente en la orilla sur del camino, a favor de la pendiente. Una parte de este está ocupada por un banco de arenisca. El resto estuvo empedrado en el pasado, pero hoy está hormigonado, excepto en la zona más alta, para favorecer tanto el acceso de vehículos de motor como la recogida de agua.

\subsection{Sistema hídrico}

La gestión del sistema hídrico de la unidad básica del sistema de riegos de monte del Coronazo se subdivide en tres partes: captación de agua, almacenamiento y su aplicación al riego. Se supone que no hay pérdidas directas relacionadas con el riego por escorrentía superficial, dado el sistema manual de aplicación del agua. Sin embargo las balsas pierden agua por evaporación directa y, al menos algunas pierden parte del agua por percolación. Estas últimas pérdidas son difíciles de cuantificar en el momento actual. En cualquier caso, hay que tener en cuenta que al estar situadas las balsas dentro del huerto, una parte del agua infiltrada puede ser captada por los sistemas radiculares de los cultivos. 


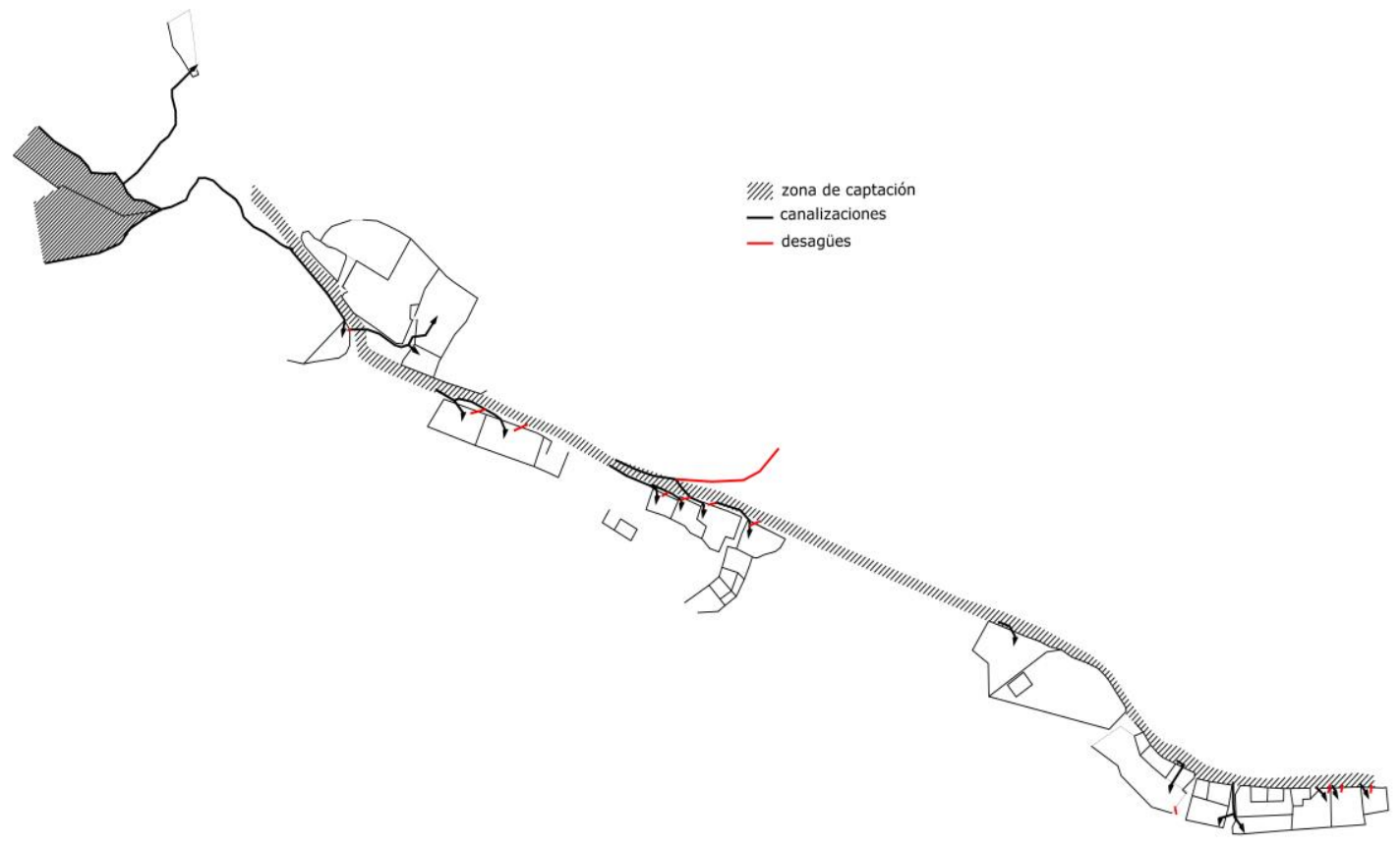

Fig. 5. Esquema de captación de agua del sistema del Coronazo

3.1.1 Captación de agua. El sistema de riego se abastece fundamentalmente del agua de lluvia recogida en el camino principal (figuras 5 y 6 ). Este tiene una longitud próxima a los 400 metros y anchura equivalente del orden $5 \mathrm{~m}$. Como ya se ha indicado, en la actualidad éste tiene muy poca permeabilidad debido al afloramiento de roca y a la base de hormigón. Esto indica un alto número de curva (NC) actual que, probablemente inferior en el antiguo empedrado.

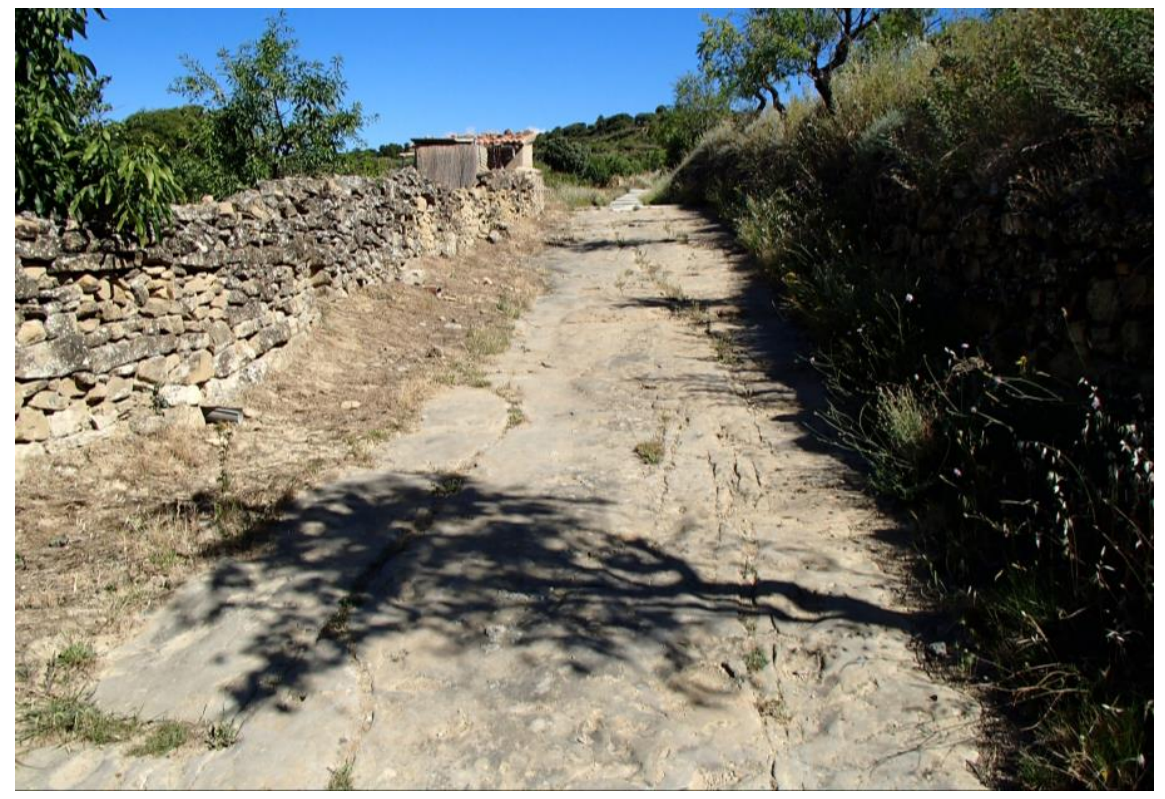

Fig. 6. Tramo en roca del camino, que forma parte del sistema de captación. 
En adición, hay un sistema de recogida de agua, tipo water harvesting, del camino antiguo de Biel, complementado por una agüera del orden de unos 100 metros de longitud, hoy sin mantenimiento. Parece que su mantenimiento se realizaba a vecinal de las casas directamente interesadas. Pudo haber sido más alargada en el pasado pero la ladera ha deslizado ligeramente. Se le estima que capta una superficie del orden de unos $2000 \mathrm{~m}^{2}$. La zona de captación, que está abancalada y que se esquematiza en la figura 7 , se utilizaba para cultivo de cereal en el pasado. En las márgenes, y después en el centro de las fajas, se cultivaron almendros. Hoy los bancales están abandonados con abundancia de aliagas y enebros.

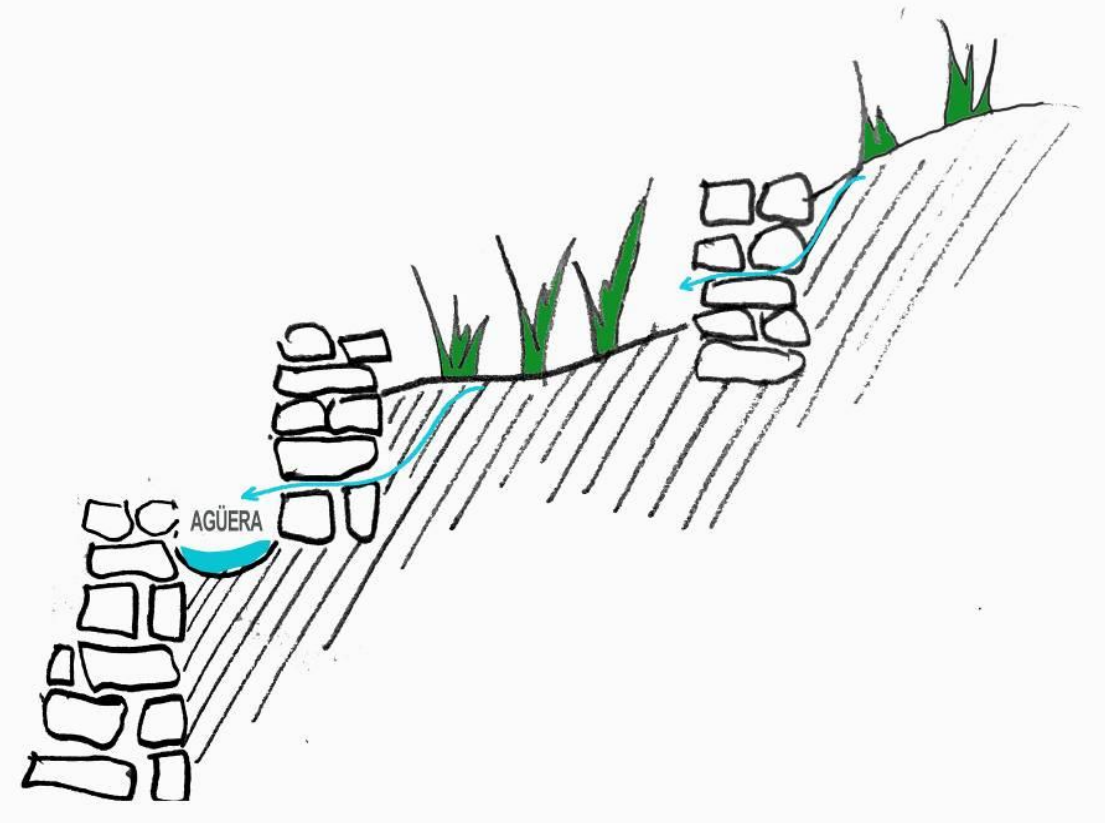

Fig. 7. Esquema captación de agua

Parte del agua de la agüera y del camino, con una pequeña acequia en tierra, suministraba a un huerto aislado y al primer grupo de huertos, vertiendo el sobrante al camino. Allí destaca el pequeño acueducto que se muestra en la figura 8 construido con tejas sobre un vallado en piedra seca. Los otros dos grupos de huertos, captaban el agua del camino mediante mínimos desviadores transversales. Como excepción, algunos huertos compartían captación (figura 9) y todavía se mantiene un pequeño partidor en tierra.

Las balsas tienen dimensiones modestas. La mayoría son rectangulares, en parte excavadas en la roca y parte en piedra seca y sin revestir (figura 10). No tienen brocal ni recubrimiento y muchas presentan una pequeña escalera para extraer el agua almacenada. En adición, parece que eran comunes los quinquinflones, aunque ya no queda ninguno. Como curiosidad, las balsas presentan abundantes ranas y, en algunas, se han encontrado tritones jaspeados.

El agua entraba por la base de los muros a las balsas que a su vez tienen un aliviadero que retorna, cuando se llenan, el agua hacia el camino (figura 11) y la siguiente captación. De este modo todas las balsas recogen agua simultáneamente, aunque hay cierta preeminencia entre las que se encuentran en cabecera de los tres subgrupos de huertos. 


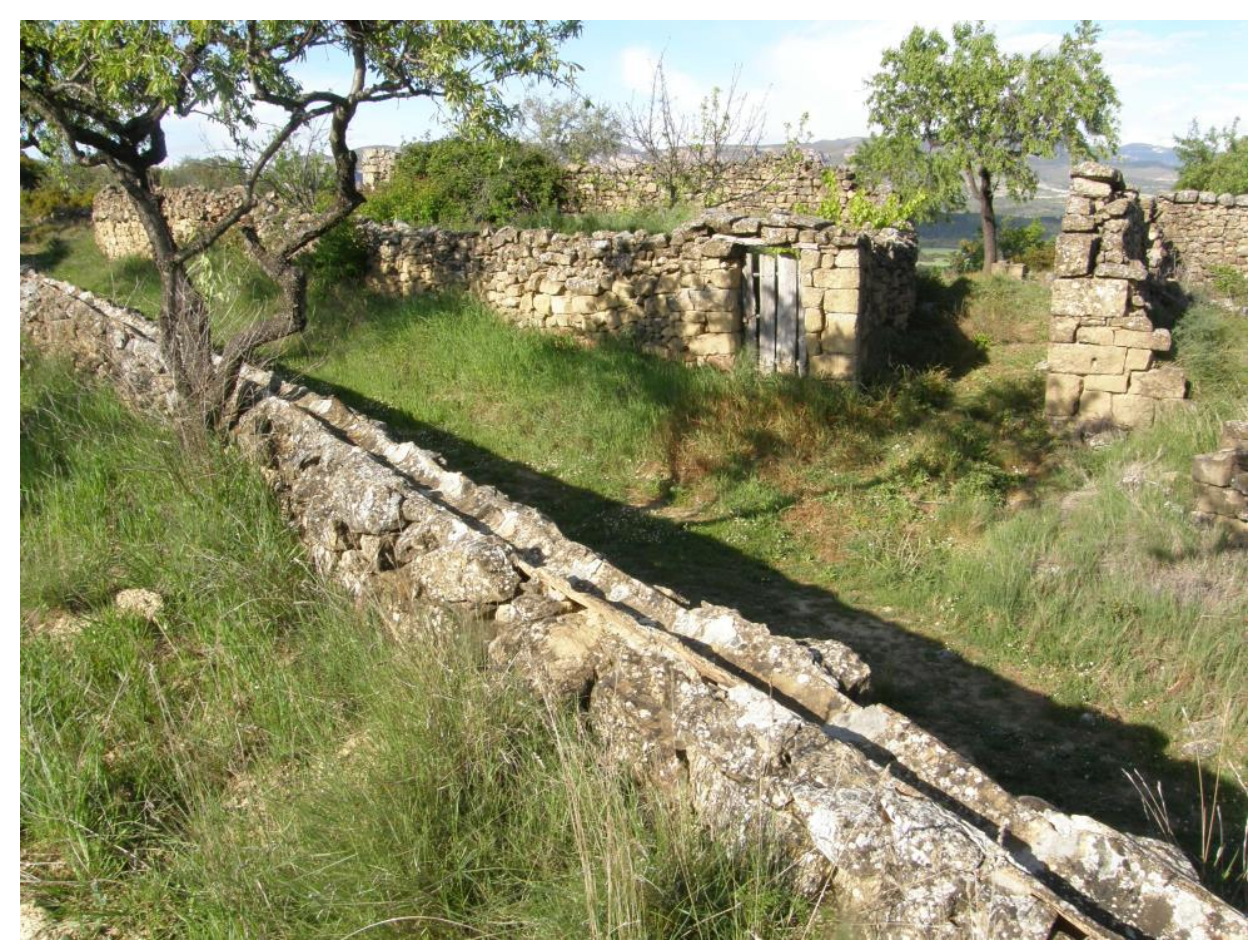

Fig. 8. Acueducto en la zona superior del sistema de riego del Coronazo.

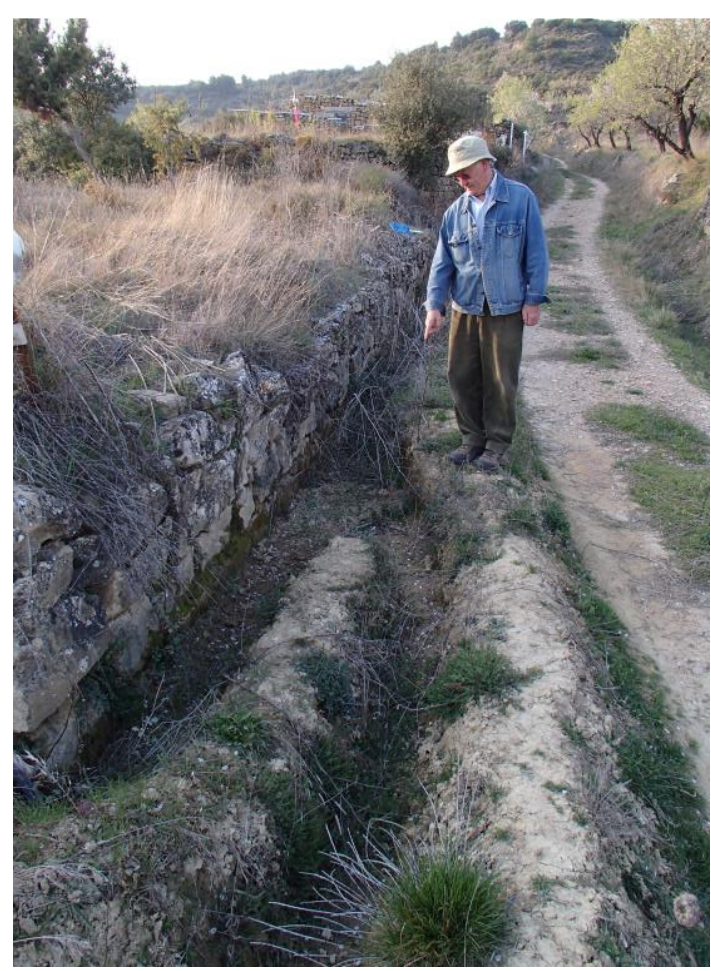

Fig. 9. Partidor de agua de escorrentía en el sistema de riego del Coronazo. 


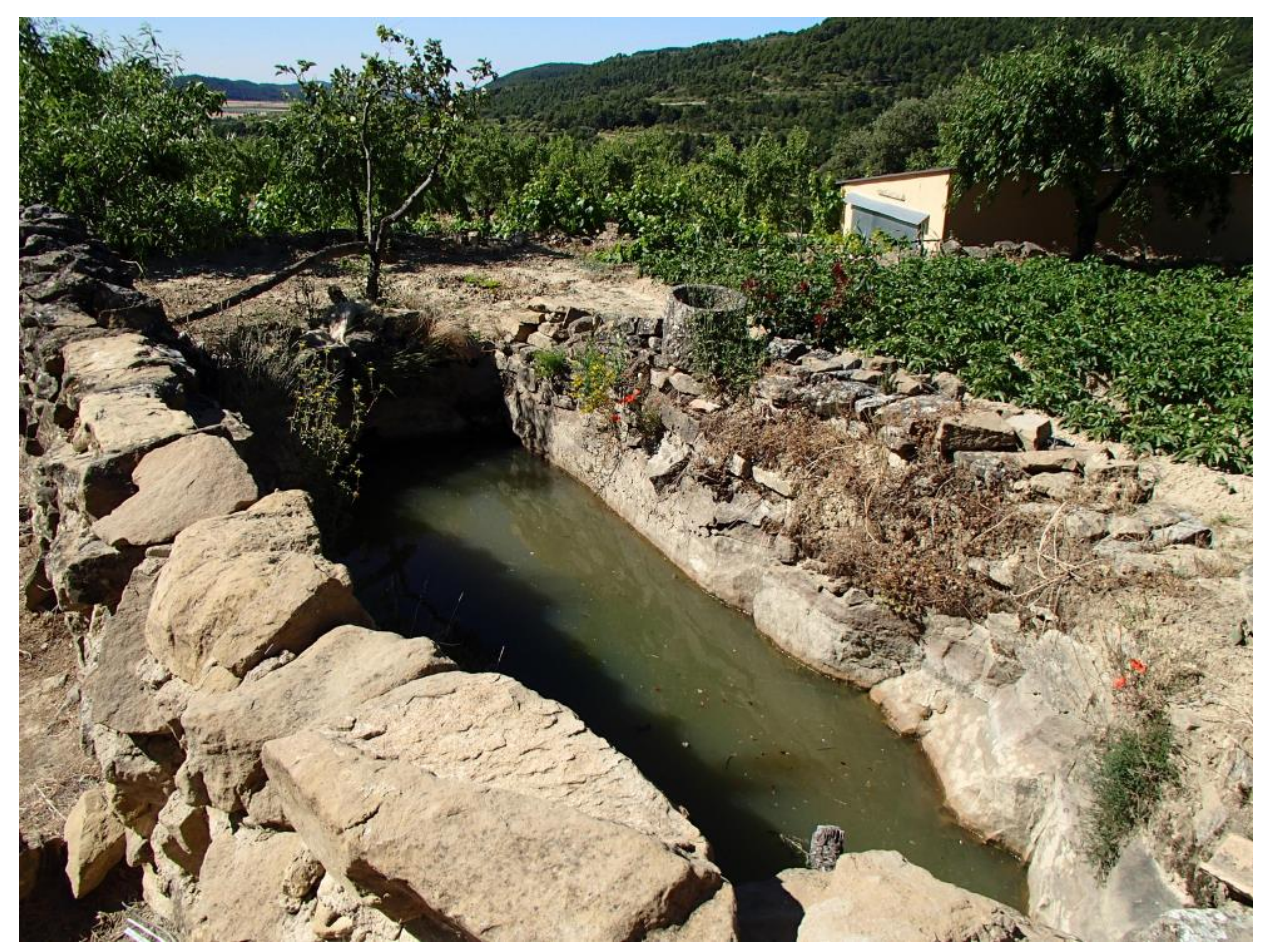

Fig. 10. Ejemplo de huerto con su balsa (recinto 19). Se observa el pilón para riego con quinquinflón.

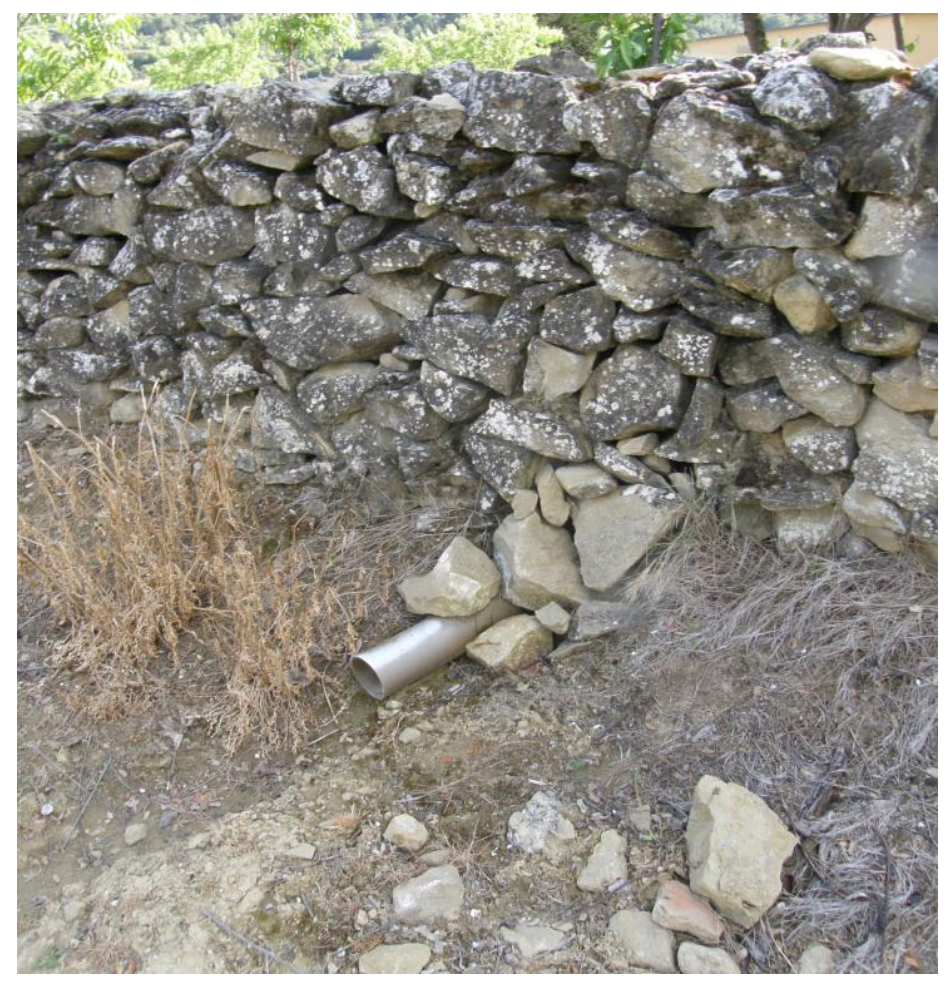

Fig. 11. Desagüe de retorno al camino desde una balsa. 
3.1.2 Necesidades hídricas y eficiencia del sistema. Con el fin de analizar la eficiencia del sistema, se ha realizado un predimensionado de las necesidades hídricas del cultivo. Se trata de una aproximación que debe de ser tomada con todas las precauciones y que necesita de trabajo de campo para su validación.

Las necesidades de riego mensuales (IN) se establecen a partir de la fórmula:

$$
\mathrm{IN}=\text { ETcrop-Pe }
$$

donde Etcrop es la necesidad hídrica del cultivo ( $\mathrm{mm} / \mathrm{mes}$ ) y Pe es la precipitación efectiva (mm/mes). Esta se estima como el $80 \%$ de la precipitación caída directamente sobre la zona cultivada de los huertos.

Evidentemente las necesidades reales deben ajustarse al desarrollo del cultivo. Se ha tomado un valor estimativo de 90 días como tiempo de crecimiento para una serie de cultivos de autoconsumo doméstico plantados estas huertas en la época de clima más extremo (primavera-verano). Los cultivos seleccionados son lechugas, tomates, pimientos, calabazas, pepinos, cebollas, judías y zanahorias en proporciones iguales.

Las necesidades de evapotranspiración se han obtenido mediante el método de Blaney-Criddle:

$$
\text { ETcrop }=\text { Kc } x \text { Eto }
$$

donde Kc es el coeficiente de cultivo y ETo la evapotranpiración de referencia.

La evapotranspiración de referencia se ha calculado según:

$$
\text { ETo }=\mathrm{p}(0.46 \text { Tmedia }+8)
$$

donde ETo $=$ evapotranspiración del cultivo de referencia $(\mathrm{mm} /$ día $)$, Tmedia $=$ Temperatura media diaria $\left({ }^{\circ} \mathrm{C}\right)$ y $\mathrm{p}=$ porcentaje medio diario anual de horas durante el día. Los datos climatológicos se han obtenido de la Estación Huesca-Aeropuerto, de la Agencia Estatal de Meteorología.

Los resultados indican una ETo del orden $500 \mathrm{~mm}$ y una precipitación eficaz del orden de $100 \mathrm{~mm}$. La diferencia $400 \mathrm{~mm}$ debe de ser aportada mediante la recogida de agua fuera de los huertos. Sobre una estimación de 0,5 hectáreas de huertos, ampliamente sobrepasada en la realidad, $400 \mathrm{~mm}$ suponen una demanda de $2000 \mathrm{~m}^{3}$ que debiera ser aportado por las escorrentías.

En principio el camino parece producir más agua que la zona de agüera. Con una superficie aproximada de $2000 \mathrm{~m}^{2}$ y una lluvia total de $100 \mathrm{~mm}$ suponen un volumen de $200 \mathrm{~m}^{3}$, que multiplicado por un factor minorizador (coeficiente de escorrentía) de 0,8 implica una recogida de $192 \mathrm{~m}^{3}$. El porcentaje recogido en la agüera será menor. A partir de un coeficiente de escorrentía de 0,2, los aproximadamente $2000 \mathrm{~m}^{2}$ de área suponen $48 \mathrm{~m}^{3}$ de agua. Haciendo el cálculo inverso, la superficie máxima de huerto no puede superar los $600 \mathrm{~m}^{2}$.

El total recogido no compensa el déficit ya señalado e indica que la superficie plantada debe de ser cuidadosamente controlada. Por otro lado, el agua recogida tiene que ser almacenada en las balsas. Estimando un total de 20 balsas, el volumen medio para almacenar es de 12 $\mathrm{m}^{3}$ por del tamaño observado. En cualquier caso, en esta estimación hay que tener en cuenta 
las pérdidas por evaporación y, sobre todo, las pérdidas por infiltración de algunas balsas, especialmente las que no están excavadas en roca.

Los cálculos sugieren, una alta eficacia del riego, que tiene una gran dependencia de las condiciones meteorológicas de cada año. También parece que el sistema está muy cerca de sus límites de aprovechamiento hídrico. Evidentemente los periodos de sequía, especialmente cuando fallan las lluvias de primavera, han tenido que afectar duramente a la productividad y por tanto a las familias.

\section{Gestión de recursos comunes}

\subsection{Un grupo humano concreto}

Detrás del sistema de riego, es evidente que se encontraba un grupo humano que lo ideó, construyó y mantuvo por sus necesidades. Hoy este grupo ha evolucionado por factores diversos que incluyen desde la emigración, temporal o definitiva, a la herencia. Pero la enumeración de las casas propietarias hace unos 50 años indica que en la mayoría de los casos se trata de familias de modesta condición radicadas en el cercano barrio alto de Santolaria. Esto se corrobora además por la arquitectura, escala y materiales de sus viviendas.

Se conoce muy poco la evolución del núcleo urbano de Santolaria, pero las fechas en los dinteles de las casas de este barrio, en torno a 1850, sugieren que el sistema de riego del Coronazo pudiera tener una edad similar, dentro de la necesidad de dar respuesta al aumento de población y sus necesidades alimentarias. Las dimensiones de los huertos indican un evidente objetivo de auto subsistencia en adición a pequeños corrales.

No se ha identificado ningún grado superior de organización. La existencia de una balsa por huerta elimina los problemas de distribución post almacenamiento, y dado que este reparto y almacenamientos e hacen en un momento de abundancia del recurso. Al mismo tiempo, la autolimitación en el tamaño de las balsas, sugiere además de un conocimiento del medio, un cierto grado de solidaridad entre los vecinos. De hecho, las entrevistas realizadas indican una buena armonía entre los cultivadores de esta zona.

A pesar de sus muy modestas dimensiones, el sistema de riegos de Monte del Coronazo presenta interesantes características por su singularidad respecto a otros sistemas de riego existentes de la zona, descritos parcialmente por Monter y Benito (1986) como por el aprovechamiento de un recurso escaso y sus instrumentos de cosecha de agua, que denotan un gran conocimiento del clima y del suelo del lugar, así como por su sistema de autogestión.

\subsection{Recurso de acervo común y habitabilidad}

La mayoría de los sistemas de recursos naturales usados por varios individuos pueden ser clasificados como recursos de acervo común. (Ostrom, 1990). Entendiendo que la gestión del recurso agua de escorrentía y su relación con los usuarios que lo comparten de forma auto organizada o incluso no organizada ha tenido a lo largo del tiempo un éxito considerable, se deduce que ésta gestión cuenta o ha contado con un nivel de regulación suficiente como para asegurar su permanencia.

De los datos históricos y socioculturales de la sociedad a la que pertenecía el grupo de usuarios del recurso, deducimos que la combinación de algunas de las variables detectadas en la 
gestión del sistema permitieron que éste fuese autogestionado y sostenible a lo largo de un considerable periodo de tiempo.

De hecho, en este sistema se encuentran la mayoría de los huertos que han sobrevivido sin auxilio de la red municipal de abastecimiento.

Las variables detectadas para el sistema del Coronazo son las siguientes:

- $\quad$ Un grupo pequeño y homogéneo de usuarios que explotaban el recurso.

- Dependían del recurso para su subsistencia.

- Tenían antecedentes culturales similares que les conducía a compartir la compren sión común de las situaciones y por tanto tenían una tasa de desencuentro baja.

- $\quad$ Contaban con un interés común que era básicamente la autosubsistencia.

- $\quad$ Existía autoconfianza mutua.

- Contaban con autonomía para establecer sus propias reglas.

La combinación de estas variables conducía a un equilibrio de los costos y los beneficios de la gestión. Éste equilibrio sería posiblemente el que llevaría a la sostenibilidad durante un período de tiempo determinado y el desequilibrio de estas variables sería el que posiblemente derivaría en el deterioro del sistema de gestión hasta prácticamente su desaparición en la actualidad. Este desequilibrio habría sido inducido por la despoblación del municipio, reduciendo la presión y por tanto demanda de eficiencia máxima del sistema, diversificando los objetivos que los usuarios tenían sobre el recurso y el cultivo que regaban. Todo ello habría derivado a un desinterés por la infraestructura y a un abandono parcial de la misma.

Si el análisis del origen del sistema es correcto, este era prácticamente la última solución ante un incremento poblacional. Por la geomorfología del municipio quedaban pocas zonas puntos para huerta dado que el riego no es posible en cualquier parte. Por tanto los habitantes del lugar, en un momento histórico de mayor presión demográfica pudieron desarrollan técnicas conocidas y observadas en otros puntos del monte, pero aquí en el Coronazo, de forma organizada y comunitaria para poder abastecerse de hortaliza y legumbre principalmente a lo largo de las épocas de verano.

La gestión del recurso desde su complejidad y sostenida en el tiempo ha permitido en parte mantener la habitabilidad de este territorio durante épocas de mayor o menor presión demográfica, sequía, enfermedad, despoblación, etc. Lo que demuestra la flexibilidad tanto del sistema técnico y su mantenimiento como de la gestión y la adaptabilidad a los cambios sociales.

\section{Conclusiones}

Este sistema hídrico fue diseñado y ejecutado de forma comunitaria y la gestión y el uso ha perdurado más de un siglo. De hecho y como ya se ha señalado, este sistema alberga a la mayoría de los huertos antiguos que han supervivido a los cambios sociales y tecnológicos de las últimas décadas. 
Así pues las infraestructuras supervivientes son partes vivas de una trama compleja compuesta por agüeras, taponeras, balsas y muros, pero también por vecinales, turnos, comunidad, auto subsistencia, confianza y autonomía, y todas estas piezas son precisas para la pervivencia del sistema.

Es evidente que quedan preguntas sin respuestas. ¿Se construyó el sistema bajo el diseño de un sistema unitario y cerrado? ¿Cuáles son los límites de crecimiento de este tipo de sistemas? ¿Se llegó al límite "normal" del recurso? El tema queda abierto a nuevos estudios, futuras líneas de investigación y otras interpretaciones.

\section{Agradecimientos}

Agradecer la paciencia de los dueños de los diversos huertos en transmitirnos sus conocimientos y en especial a Donato Sánchez, de casa Leon de Metro (Señá Modesta), Benito Arbués, de casa Pito; Miguel Posa, Antonio y María Pilar Rubiol y Elba Alastuey de casa Severino, Ramón Alastuey, de casa Gariseta; Pedro Cañas; Luis Maleta; Rosa y José Ramón Arbués de casa Marcela; José María Liso, de casa Polito; José Arbués de casa Esteban de Morán. Carlos Mur hizo el detallado trabajo de topografía.

\section{Referencias}

Cuchí, J.A. (2006): Anotaciones sobre la distribución de agua en los sistemas de riego tradicionales de la zona occidental de la Hoya de Huesca. Anales de la Fundación Joaquín Costa. 2223: 5-46.

Evanari, M., Shanan, L., Tadmor, N.H. (1971): The Negev. The Challenge of a Desert. Harvard University press, Cambridge, MA, USA.

Frot, E., van Wesemael, B., Benet, A.S., House M.A. (2008). Water harvesting potential in function of hillslope characteristics: A case study from the Sierra de Gador (Almeria province, south-east Spain. Journal of Arid Environments 72: 1213-1231.

Glick, T.F. (1968): Levels and Levelers: Surveying Irrigation Canals in Medieval Valencia, Technology and Culture, 9:165-180.

Laliena, C. (ed). (1994): Agua y proceso social. Siete estudios sobre el regadío en Huesca. Instituto de Estudios Altoaragoneses. Huesca.

Monter, M. J., Benito L. (1986). La acequia de Albalate en el siglo XV: aproximación al estudio del regadío medieval en el valle del Cinca. Argensola 100: 167-176.

Ngigi, S.N. (2003): What is the limit of up-scaling rainwater harvesting in a river basin? Physics and Chemistry of the Earth 28: 943-956.

Ostrom E. (1990): Governing the commons: the evolution of institutions for collective action, New York, Cambridge University Press.

Oweis, T., Hachum, A., Kijne, J. (1999). Water harvesting and supplemental irrigation for improved water use efficiency in the dry areas. SWIM Paper 7, International Water Management. Institute, Colombo, Sri Lanka.

Piedrafita, E. (1995): Infraestructura económica de los concejos de las Cinco Villas: regadíos, molinos y hornos (siglos XII-XIV). Aragón en la Edad Media, 12: 29-60. 